\title{
LA PROBLEMÁTICA DE LA PROTECCIÓN DEL DERECHO A LA SALUD DE LAS PERSONAS MIGRANTES EN TRÁNSITO POR MÉXICO
}

\author{
Eduardo Acosta Lobatos ${ }^{10}$ \\ Ma. Auxiliadora Moreno Valenzuela ${ }^{11}$
}

Sumario: I. Introducción. II. La migración en tránsito migratorio y su inclusión en el Programa Vete Sano Regresa Sano. Conclusión.

\section{Resumen.}

En México la atención a la salud de las personas migrantes que intentan cruzar la frontera rumbo a los Estados Unidos de Norteamérica, tiene como plataforma, entre otros, el "Programa Vete Sano, Regresa Sano", PVSRS. En este trabajo se buscó reflexionar sobre cuáles son las condiciones que posibilita el programa precitado, para el ejercicio del derecho a la salud por parte de las personas migrantes en tránsito por nuestro país, buscando identificar en un primer momento, como dicho programa incorpora a este sector de la población a partir de su problemática de salud, para posteriormente, identificar la acciones de promoción y atención a la salud que contempla y la eficacia de algunas de ellas.

Palabras clave: Migrantes en tránsito, derecho a la salud

\section{Abstract}

In Mexico, the healthcare provided to migrants who try to cross the border towards the United States of America, is based, amongst others, on programs such as "leave healthy, return healthy". This paper reflects upon the conditions on which this program enables the excercise of the right to health for migrants in transit through Mexico, with the objective to identify how said program

\footnotetext{
${ }^{10}$ Estudiante de la licenciatura en Derecho del Departamento de Derecho de la DCS UNISON URC

${ }^{11}$ Profesora Investigadora de Tiempo Completo adscrita al Departamento de Derecho de la DCS URC UNISON
} 
incorporates this population sector, taking into consideration their health issues, as well as to identify the promotion actions and contemplated healthcare and their effectiveness.

Keywords: Migrants in transit, right to health.

\section{I.- Introducción}

El derecho a la salud se encuentra contemplado en el artículo $4^{\circ}$ de la Constitución Política de los Estados Unidos Mexicanos, derivado de ello el Estado está obligado a formular una política pública encaminada a posibilitar el acceso a la salud de los titulares de dicho derecho. Las persona migrantes en tránsito por México son titulares de todos los derechos establecidos en dicho documento constitucional, y en los tratados relativos firmados por nuestro país, así como en la legislación secundaria aplicable.

La Ley de Migración reconoce el derecho a la salud de las personas migrantes en México, independientemente de su condición legal. ${ }^{12}$ La misma Ley en su artículo 27 fracción primera, señala que será la Secretaría de Salud la encargada de “...promover en coordinación con las autoridades sanitarias de los diferentes niveles de gobierno, la prestación de servicios de salud que se otorgue a los extranjeros, se brinde sin importar su situación migratoria y conforme a las disposiciones jurídicas aplicables."

${ }^{12}$ El Artículo 8 de la Ley de Migración establece que "Los migrantes tendrán derecho a recibir cualquier tipo de atención médica, provista por los sectores público y privado, independientemente de su situación migratoria, conforme a las disposiciones legales y reglamentarias aplicables. Los migrantes independientemente de su situación migratoria, tendrán derecho a recibir de manera gratuita y sin restricción alguna, cualquier tipo de atención médica urgente que resulte necesaria para preservar su vida.

En la prestación de servicios educativos y médicos, ningún acto administrativo establecerá restricciones al extranjero, mayores a las establecidas de manera general para los mexicanos. 
En México la atención a la salud de las personas migrantes que intentan cruzar la frontera rumbo a los Estados Unidos de Norteamérica, tiene como plataforma, entre otros, el Programa Vete Sano, Regresa Sano", PVSRS.Dicho documento señala que "Anualmente migran hacia el exterior de nuestro país 1.4 millones de personas, siendo el 98\% hacia los EUA, con una pérdida neta anual de 396,000."13 Este documento reconoce, además, que la condición de indocumentados, independientemente de la composición interna de los grupos de migrantes internacionales, es la que coloca a estas personas en situación de vulnerabilidad, “...dado que más de las dos terceras partes de los migrantes, no cuentan con sistemas de atención en el lugar destino."14

En este trabajo se busca reflexionar sobre cuáles son las condiciones que posibilita el programa vete sano regresa sano, para el ejercicio del derecho a la salud por parte de las personas migrantes en tránsito por nuestro país, buscando identificar en un primer momento, la forma en que dicho programa incorpora a este sector de la población a partir de su problemática de salud, para posteriormente, identificar la acciones de promoción y atención a la salud que contempla y la eficacia de algunas de ellas.

\section{II.- La migración en tránsito migratorio y su inclusión en el Programa Vete Sano, Regresa Sano", PVSRS.}

El programa precitado, se formuló con orientación a la población migrante, con la finalidad de otorgar una protección a la salud por considerarlos como grupos en condición de vulnerabilidad planteándose como tarea el reforzamiento de "los servicios de promoción de la salud en poblaciones indígenas marginadas y hacia los migrantes en los lugares de origen, tránsito y destino" ${ }^{15}$

\footnotetext{
13 Programa de Acción en Especifico, Vete Sano Regresa Sano 2007- 2012, Secretaria de Salud, México. Primera edición, en

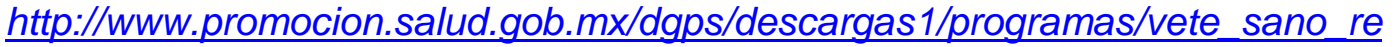
gresa sano.pdf [fecha de consulta octubre de 2013]

14 Idem

${ }^{15}$ Idem. P. 19
} 
De igual forma, en el "Programa de acción específico, vete sano, regresa sano" ${ }^{16}$ documento complementario del $\mathrm{PVSRS}^{17}$ se señala como misión principal del mismo, "la reducción de la vulnerabilidad de los migrantes estableciendo líneas de acción prioritaria la promoción de la salud en los sitios de origen, tránsito y destino."18

En el primero de estos documentos, se clasifican a las enfermedades de las personas migrantes como: enfermedades que se adquieren desde los lugares de origen y enfermedades que se adquieren en los lugares de destino. Entre las enfermedades de origen se encuentran, las infecciones respiratorias agudas, infecciones intestinales, infecciones de vías urinarias, ulceras, gastritis, hipertensión arterial, diabetes, entre otras; y en cuanto a las enfermedades de las comunidades de destino, se señalan a la diabetes, obesidad, hipertensión, tuberculosis, stress - depresión, entre otras.

Sin embargo ambos documentos omiten caracterizar las enfermedades del tránsito migratorio, a pesar de que en ellos se reconoce que las personas migrantes por ser una población en condiciones de vulnerabilidad están expuestos a los riesgos que tiene el circuito migratorio, tampoco señalan como influyen estos riesgos en la profundización de las enfermedades que ya se traen de las comunidades de origen, es decir las enfermedades del transito migratorio podrían ser una prolongación de las enfermedades de origen pero que debido a las características de la movilidad muchas veces clandestinas se agudizan tanto por las condiciones del viaje que pueden ser desde falta de acceso a alimentos y agua potable hasta el stress provocado por los altos índices de violencia que generalmente acompañan el transito migratorio.

16 Programa de Acción: Migrantes "Vete Sano Regresa Sano" Versión Preliminar, en http://www.salud.gob.mx/docprog/estrategia_1/migrantes.pdf. Fecha de consulta: octubre de 2013.

17 Las siglas PVSRS significan: Programa Vete Sano Regresa Sano

18 Programa de Acción en Especifico, Vete Sano Regresa Sano 2007- 2012, Secretaria de Salud, México. Primera edición, disponible en

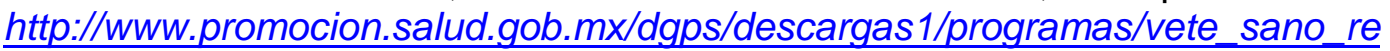
gresa sano.pdf fecha de consulta octubre de 2013. P. 7 
EI PVSRS se basa en ocho estrategias para la protección a la salud de las personas migrantes, entre las que se encuentran, la de fortalecer la coordinación sectorial de las instituciones de salud, el diagnosticar la situación de la salud de las personas migrantes, y la utilización del sistema de atención de salud médica existente, entre otras.

En este mismo sentido en una encuesta derivada del proyecto "uso y acceso a servicios de salud de los migrantes guanajuatenses 2004-2005", aplicada por parte de la EMIF en los estados de Tijuana, Nuevo Laredo y Ciudad Juárez a las personas migrantes del sur de México, para documentar la condiciones de salud que las personas migrantes presentan, arrojó entre los resultados de la aplicación de dicha encuesta que los programas de salud que tiene el Estado Mexicano y las instituciones obligadas de brindarlo resultaron desconocidos para los migrantes que transitan por el territorio mexicano rumbo hacia EEUU.

"Los programas de salud o instituciones en México que les pueden brindar atención médica a estas personas migrantes resultaron prácticamente desconocidos; sólo el 2.3\% de las y los migrantes provenientes de EUA conocían algún programa de salud en México, proporción que ascendía al 3.4\% de los que procedían del sur de México."19

Esta situación de desconocimiento de las formas y mecanismos por parte de las personas migrantes en tránsito para acceder a los servicios de salud cuestiona la eficacia de la operatización de los programas orientados no solo a la promoción de la salud de este sector en condición de vulnerabilidad, sino también de la atención de estas personas a fin de dar cumplimiento a las obligaciones del Estado Mexicano a través de sus instituciones de atención médica durante todo el circuito migratorio y no tan solo cuando los migrantes son puestas a disposición de algunas autoridades como lo son el Instituto Nacional de Migración (INM), grupos Betas, alojamiento en estaciones

19 Herrera, Carmen, "Factores Condicionantes del Acceso a los Servicios de Salud de las Personas Migrantes en Transito". El caso de Tijuana. Agosto 2010, México. En

http://www.flacsoandes.org/dspace/bitstream/10469/3908/1/TFLACSO-042010MCHB.pdf 
migratorias en el programa de repatriación humana, incluso asistencia por parte de miembros de la organización civil que en la mayoría de los casos no cuentan con recursos suficientes para proporcionar una atención medica eficaz y necesaria, [...]"el consultorio médico de VSRS brinda, en un horario acotado, atención básica en salud [...] En las oficinas del Grupo Beta, las personas tienen la opción de hacer una llamada telefónica gratuita y se les entrega una sopa instantánea marucha ${ }^{20}[\ldots]$

Así de igual manera el programa vete sano regresa sano (PVSRS), se ha adherido a otros organismos que han tenido interés en la atención de la salud, tal es el caso de Médicos del Mundo, donde el eje motor inicial de este organismo es brindar servicios de salud a los migrantes que intentan cruzar por la frontera norte, dirigido en especial a la población migrante que padece la enfermedad VIH/SIDA, donde la principal tarea de este organismo es la participación con la asignación de un documento que posibilitara el acceso a la salud de los migrantes en tránsito de manera gratuita, llamado este el "pasaporte de salud" sin embargo las expectativas que tenían este organismo no fueron cumplidas puesto el Programa Vete Sano Regresa Sano no contaba con recursos económicos para llevar a la practica este servicio: primero porque dicho programa, era conocido por pocas personas que trabajan en instituciones de salud, y segundo, por desconocimiento total del "pasaporte de salud" del mismo personal de instituciones médicas y por los mismo migrantes ${ }^{21}$

"Precisamente, respecto al acceso a los servicios de salud, Médicos del Mundo halló que, entre las personas migrantes que habían enfermado, $17 \%$ no tuvieron acceso a estos servicios. Las razones aducidas fueron: $27 \%$ por falta de recursos económicos, $21 \%$ no sabían a dónde acudir, 13\% porque se requerían muchos papeles para la atención médica, $11 \%$ por temor a ser rechazado o mal atendido y $28 \%$ no consideraron necesario atenderse"

\footnotetext{
${ }^{20}$ Idem

${ }^{21}$ Ibidem, p. 78.
} 
En este mismo sentido Médicos del Mundo señala que el Programa Vete Sano Regresa Sano es un programa que no puede llevar a la practica las encomiendas en él propuestas para la atención de la salud de las personas migrantes, puesto que un factor que interviene y que hace que imposibilite el ejercicio de este derecho, es lo que tiene que ver con la escasez de los recursos presupuestarios por parte del gobierno para impulsar este programa, señalando que la participación del gobierno queda solamente en buenas intenciones y por tanto las acciones de dicho programa quedan plasmadas en un documento que carece de eficacia.

"Casi ninguna campaña pública de información y si bien existen documentos de información específicamente diseñados para los migrantes, esos documentos no son distribuidos en los lugares donde se encuentran los migrantes. Ninguna acción de prevención ha sido desarrollada todavía. Después de tres años de presencia y de actividades en Tijuana y Mexicali, podemos afirmar que ninguno de los 1535 migrantes vistos en consultas conocía la existencia de tal Programa y solo algunos de los servidores públicos de los Servicios de Salud"

De igual manera Médicos del Mundo, hace énfasis en la importancia de que el Programa Vete Sano, Regresa Sano PVSRS, tenga un verdadero interés en hacer posible y que garantice el acceso a la salud de los y las personas migrantes, brindando "acceso gratuito a la salud, consultas, medicamentos, tratamiento, exámenes complementarios para reducir los riesgos sanitarios físicos y psicológicos de la población migrante prevención de enfermedades infectocontagiosas y de transmisión sexual; seguimiento a las personas que llegan diagnosticadas y con tratamiento médico desde EUA"22

22 Ibidem, p. 79 


\section{III.-Conclusiones}

El programa vete sano regresa sano se basa principalmente en la promoción de la salud de la población migrante en los lugares de origen, tránsito y destino, señalando en él las enfermedades que las y los migrantes presentan en los lugares de origen y de destino omitiendo señalar cuales son las enfermedades que los migrantes presentan durante el transito migratorio.

En este mismo sentido el derecho a la salud de los y las personas migrantes que transitan por México queda limitado por factores que trae aparejado el circuito migratorio, tales como las redes de tráfico de personas, la inseguridad social, abuso de autoridades, burocratismo gestión pública ineficaz y falta de presupuesto para los programas que apoyen al sector migrante.

Así de igual manera el Programa Vete Sano, Regresa Sano, PVSRS no cuenta con los recursos suficientes por parte del Estado Mexicano para hacer efectivo el derecho a la salud quedando solamente en enunciados teóricos que no permiten llevar a la práctica las acciones planteadas.

El Programa Vete Sano, Regresa Sano, PVSRS imposibilita el ejercicio del derecho a la salud si no cuentan la dicha atención médica en los lugares y rutas de tránsito migratorio, donde precisamente, se presentan los principales riesgos a la salud física y emocional de las personas en tránsito por nuestro país. Estos riesgos se encuentran asociados a la pobreza y la inequidad que están personas viven en sus lugares de origen, condiciones que los hace proclives,-una vez que se ponen en camino-, a ser sujetos de más violencia en y durante el tránsito migratorio.

\section{Referencias Bibliográficas y Webgraficas :}

Programa de Acción en Especifico, Vete Sano Regresa Sano 2007- 2012, Secretaria de Salud, México. Primera edición, en http://www.promocion.salud.gob.mx/dgps/descargas1/programas/vete_sano_re gresa_sano.pdf fecha de consulta octubre de 2013. 
Programa de Acción: Migrantes "Vete Sano Regresa Sano" Versión Preliminar, en http://www.salud.gob.mx/docprog/estrategia_1/migrantes.pdf.[fecha de consulta: octubre de 2013]

Programa de Acción en Especifico, Vete Sano Regresa Sano 2007- 2012, Secretaria de Salud, México. Primera edición, disponible en http://www.promocion.salud.gob.mx/dgps/descargas1/programas/vete_sano_re gresa_sano.pdf f[echa de consulta octubre de 2013]

Programa de Acción: Migrantes "Vete Sano Regresa Sano" Versión Preliminar, en http://www.salud.gob.mx/docprog/estrategia_1/migrantes.pdf.[fecha de consulta: octubre de 2013]

Programa de Acción en Especifico, Vete Sano Regresa Sano 2007- 2012, Secretaria de Salud, México. Primera edición, disponible en http://www.promocion.salud.gob.mx/dgps/descargas1/programas/vete_sano_re gresa_sano.pdf [fecha de consulta octubre de 2013]

Herrera, Carmen, "Factores Condicionantes del Acceso a los Servicios de Salud de las Personas Migrantes en Transito". El caso de Tijuana. Agosto 2010, México. En

http://www.flacsoandes.org/dspace/bitstream/10469/3908/1/TFLACSO-04-

2010MCHB.pdf 